\title{
Highly Stereoselective Radical Carbonylations of gem- Dihalocyclopropnane Derivatives with CO
}

Yoshinori Nishii, ${ }^{\star}$ Takao Nagano, Hideki Gotoh, ${ }^{\dagger}$ Ryohei Nagase, ${ }^{\dagger}$ Jiro Motoyoshiya, Hiromu Aoyama, Yoo Tanabe ${ }^{t *}$

Department of Chemistry, Faculty of Textile Science and Technology, Shinshu University, Ueda, Nagano 3868567, Japan. Department of Chemistry, School of Science and Technology, Kwansei Gakuin University, Sanda, Hyogo 669-1337,Japan.

General Information

Experimental Procedure

Spectra data (NMR Chart)

\section{Table of Contents}

\section{$\mathrm{S} 1$}

$\mathrm{S} 1-\mathrm{S} 13$

S14-S44

\section{Supporting Information}

General: All reactions were carried out in oven-dried glassware and stainless steel autoclave. Benzene was distilled from sodium. Column chromatography was performed with silica gel Merck 60 (70-230 mesh ASTM). TLC analysis was performed on $0.25 \mathrm{~mm}$ Silica gel Merck $60 \mathrm{~F}_{254}$ plates. NMR spectra were recorded on $400 \mathrm{MHz}$ spectrometer, operating at 400 or $300 \mathrm{MHz}$ for ${ }^{1} \mathrm{H}$ NMR and 100 or $75 \mathrm{MHz}$ for ${ }^{13} \mathrm{C}$ NMR. Chemical shifts (_ ppm) in $\mathrm{CDCl}_{3}$ were reported downfield from TMS $(=0)$ for ${ }^{1} \mathrm{H}$ NMR. For ${ }^{13} \mathrm{C}$ NMR, chemical shifts were reported in the scale relative to $\mathrm{CDCl}_{3}(77.00 \mathrm{ppm})$ as an internal reference. Mass spectra were obtained by electron ionization (EI).

Preparation of gem-dihalocyclopropanes. gem-Dihalocyclopropanes $\mathbf{1 a}-\mathbf{1 j}$ were prepared by phase transfer methods as previously described..$^{1-10}$

1,1-Dichloro-2-phenylcyclopropane (1a) ${ }^{2}$

1,1,-Dichloro-2-hexylcyclopropane (1b) ${ }^{3}$

(2,2-Dichlorocyclopropyl)methanol (1c) ${ }^{4}$

(2,2-Dichloro-1-methylcyclopropyl)methanol (1d) ${ }^{5}$

[(1S*3: $\left.S^{*}\right)$-2,2-Dichloro-3-phenylcyclopropyl]methanol $(1 \mathrm{e})^{6}$

[(1S $\left.S^{*}, 3 R^{*}\right)$-2,2-Dichloro-1-methyl-3-phenylcyclopropyl]methanol (1f) ${ }^{7}$

7,7-Dichlorobicyclo[4.1.0]heptane (1g) ${ }^{1,2}$

9,9-Dichlorobicyclo[6,1,0]nonane $(1 \mathrm{~h})^{8}$

7,7-Dibromobicyclo[4.1.0]heptane (1i) ${ }^{9}$

cis- and trans-1-Bromo-1-chloro-2-phenylcyclopropane $(\mathbf{1 j}){ }^{10}($ cis/trans $=1 / 1)$

1) Makosza, M.; Wawrzyniewicz, M.; Tetrahedron Lett. 1969, 4659.

2) Chien, C.-T.; Tsai, C.-C.; Tsai, C.-H.; Chang, T.-Y.; Tsai, P.-K.; Wang, Y.-C.; Yan, T.-H. J. Org. Chem. 2006, $71,4324$.

3) Tarner, D. D.; Zhang, L. Hu, L. Q.; Kandanarachchi, P. J. Org. Chem. 1996, 61, 6818.

4) Olivo, H. F.; Velazque, F.; Trevisan H. C. Org. Lett. 2000, 2, 4055. 
5) Tanabe, Y.; Seko, S.; Nishii, Y.; Yoshida, T.; Utumi, N.; Suzukamo, G. J. Chem. Soc., Perkin Trans. 1, $1996,2157$.

6) Nishii, Y.; Tanabe, Y. J. Chem. Soc., Perkin Trans. 1, 1997, 477.

7) Nishii, Y.; Wakimuura, K.; Tsuchiya, T.; Nakamura, S.; Tanabe, Y. J. Chem. Soc., Perkin Trans. 1, $1996,1243$.

8) Ghiaci, M.; Sedaghat, M. E.; Kalbasi, R. J.; Abbaspur, A. Tetrahedron, 2005, 61, 5529.

9) Inoue, A.; Kondo, J.; Shinokubo, H.; Oshima, K. Chem. Eur. J. 2002, 8, 1730.

10) Reyne, F.; Weagell, B.; Brun, P. Bull. Chem. Soc. Jpn. 1995, 68, 1162.

cis- and trans-1-Bromo-1-chloro-2-hexylcyclopropane (1k) $($ cis/trans $=1 / 1)$

$\mathrm{C}_{6} \mathrm{H}_{13} \quad$ Chlorodibromomethane $(1.15 \mathrm{ml}, 13.4 \mathrm{mmol})$ was added to a stirred suspension of potassium $t$-butoxide $(1.50 \mathrm{~g}, 13.4 \mathrm{mmol})$ and 1-octene $(1.00 \mathrm{~g}, 8.91 \mathrm{mmol})$ in hexane

$\mathrm{Br} \quad(18 \mathrm{ml})$ at $0-5{ }^{\circ} \mathrm{C}$. After being stirred at the same temperature for $6 \mathrm{~h}$, the mixture was

$1 \mathrm{k}$ diluted with water $(50 \mathrm{~mL})$ and extracted with $\mathrm{Et}_{2} \mathrm{O}(40 \mathrm{~mL} \times 2)$. The organic phase was washed with water and brine, dried $\left(\mathrm{Na}_{2} \mathrm{SO}_{4}\right)$, and concentrated. The obtained crude oil was purified by $\mathrm{SiO}_{2}$-column chromatography (hexane) to give the product $\mathbf{1} \mathbf{k}(1 / 1$ mixture of diastereoisomers, 1.44 g, 67\%). 1k: colorless oil; ${ }^{1} \mathrm{H}$ NMR (400 MHz, $\left.\mathrm{CDCl}_{3}\right) \delta 0.88$ (t, $\left.J=7.1 \mathrm{~Hz}, 3 \mathrm{H}\right), 1.04-1.17$ $(\mathrm{m}, 1 \mathrm{H}), 1.25-1.39(\mathrm{~m}, 6 \mathrm{H}), 1.41-1.52(\mathrm{~m}, 3 \mathrm{H}), 1.55-1.71(\mathrm{~m}, 3 \mathrm{H}) ;{ }^{13} \mathrm{C} \mathrm{NMR}\left(400 \mathrm{MHz}, \mathrm{CDCl}_{3}\right) \delta$ 14.1, 22.6, 27.6, 27.9, 28.4, 28.5, 28.9, 30.3, 30.9, 31.6, 31.7, 32.6, 45.4, 48.1; IR (neat) 2957, 2927, 1466, $1113 \mathrm{~cm}^{-1}$; HRMS (EI) calcd for $\mathrm{C}_{9} \mathrm{H}_{16} \mathrm{BrCl}\left(\mathrm{M}^{+}\right)$238.0124, found 238.0128.

exo- and endo-1-Bromo-1-chlorobicyclo[4.1.0]heptane $(1 \mathrm{ll})^{11}($ exo/endo $=1 / 1)$ exo- and endo-1-Bromo-1-chlorobicyclo[6.1.0]nonane $(1 \mathrm{~m})^{11}($ exo/endo $=1 / 1)$

11) Seyferth, D.; Haas, C. K.; Dagani, D. J. Organomet. Chem. 1976, 104, 9.

[ $\left(1 S^{*}, 2 S^{*}\right)$ - and $\left(1 S^{*}, 2 R^{*}\right)$-2-Bromo-2-chloro-1-methylcyclopropyl]methanol (1n) (cis/trans $\left.=1 / 1\right)$

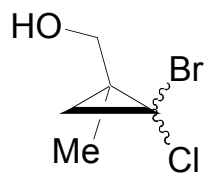

1n

A mixture of 2-methyl-2-propen-1-ol (700 mg, $9.70 \mathrm{mmol}), 3$,4-dihydro-2 $\mathrm{H}$-pyran (980 mg, $11.7 \mathrm{mmol})$, and a little amount of $p$-toluenesulfonic acid in $\mathrm{Et}_{2} \mathrm{O}(20 \mathrm{~mL})$ was stored at $\mathrm{rt}$ for $12 \mathrm{~h}$. sat. $\mathrm{NaHCO}_{3}$ aqueous solution was added to mixture, which was extracted with $\mathrm{Et}_{2} \mathrm{O}(30 \mathrm{~mL} \times 2)$. The organic phase was washed with water and brine, dried $\left(\mathrm{Na}_{2} \mathrm{SO}_{4}\right)$ and concentrated to give $1.50 \mathrm{~g}$ of the crude tetrahydropyranyl (THP) ether. Chlorodibromomethane $(4.00 \mathrm{~g}, 19.2 \mathrm{mmol})$ was added to a vigorously stirred mixture of crude THP ether and potassium $t$-butoxide $(1.62 \mathrm{~g}, 19.2 \mathrm{mmol})$ in hexane $(20 \mathrm{~mL})$ at $0-5{ }^{\circ} \mathrm{C}$. After being stirred for $18 \mathrm{~h}$ at the same temperature, the mixture was diluted with water $(30 \mathrm{~mL})$ and extracted with $\mathrm{Et}_{2} \mathrm{O}(20 \mathrm{~mL} \times 2)$. The combined extracts were washed with water, brine, dried $\left(\mathrm{Na}_{2} \mathrm{SO}_{4}\right)$, and concentrated. $\mathrm{MeOH}(20 \mathrm{~mL})$ and a little $p$-toluenesulfonic acid were added to the residue which, after being stored over night, was treated with sat. $\mathrm{NaHCO}_{3}$ aqueous solution, and then evaporated to give a residue. This was extracted with $\mathrm{Et}_{2} \mathrm{O}(20 \mathrm{~mL} \times 2)$. The extract was washed with water and brine, and concentrated to give a crude oil. The obtained crude oil was purified by $\mathrm{SiO}_{2}$-column chromatography (hexane/AcOEt $=5 / 1)$ to give the product $\mathbf{1 n}(1 / 1$ mixture of diastereoisomers, 1.13 g, 59\%). 1n: colorless oil; ${ }^{1} \mathrm{H}$ NMR $\left(400 \mathrm{MHz}, \mathrm{CDCl}_{3}\right) \delta 1.36(\mathrm{t}, J=7.6 \mathrm{~Hz}, 1 \mathrm{H}), 1.48(\mathrm{~s}, 3 \mathrm{H} \mathrm{x}$ 1/2), $1.50(\mathrm{~s}, 3 \mathrm{H} \times 1 / 2), 1.51(\mathrm{t}, J=7.6 \mathrm{~Hz}, 1 \mathrm{H}), 2.10(\mathrm{brs}, 1 \mathrm{H}), 3.66(\mathrm{~d}, J=11.9 \mathrm{~Hz}, 1 \mathrm{H} \mathrm{x} 1 / 2), 3.71$ $(\mathrm{d}, J=11.9 \mathrm{~Hz}, 1 \mathrm{H} \times 1 / 2), 3.83$ (d, $J=11.9 \mathrm{~Hz}, 1 \mathrm{H} \times 1 / 2), 3.85(\mathrm{~d}, J=11.9 \mathrm{~Hz}, 1 \mathrm{H} \mathrm{x} 1 / 2) ;{ }^{13} \mathrm{C} \mathrm{NMR}$ 
(400 MHz, $\left.\mathrm{CDCl}_{3}\right) \delta 17.7,20.9,31.6,31.7,31.9,31.9,51.8,52.1,67.5,70.1$; IR (neat) 3356, 1037, 729, $486 \mathrm{~cm}^{-1}$; HRMS (EI) calcd for $\mathrm{C}_{5} \mathrm{H}_{8}{ }^{81} \mathrm{BrClO}\left(\mathrm{M}^{+}\right)$199.9447, found 199.9456.

[(1 $\left.1 S^{*}, 2 S^{*}, 3 R^{*}\right)-$ and $\left(1 S^{*}, 2 R^{*}, 3 R^{*}\right)-2$-Bromo-2-chloro-1-methyl-3-phenylcyclopropyl]methanol (10)

(mixture of diastereoisomers: cis/trans $=1 / 1)$

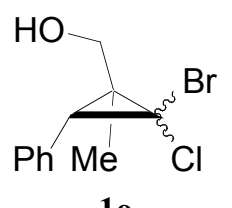

Following the procedure for the preparation of $\mathbf{1 n}$, the reaction of (E)-2-methyl- 3phenylprop-2-en-3-ol $(1.00 \mathrm{~g}, 6.70 \mathrm{mmol})$ gave the product $10(1 / 1$ mixture of diastereoisomers, $818 \mathrm{mg}, 44 \%) . \quad 10:{ }^{1} \mathrm{H} \mathrm{NMR}\left(400 \mathrm{MHz}, \mathrm{CDCl}_{3}\right) \delta 1.30(\mathrm{~s}, 3 \mathrm{H} \mathrm{x}$ 10 1/2), 1.34 (s, $3 \mathrm{H} \times 1 / 2), 1.55$ (brs, 1H), 2.63 (s, $1 \mathrm{H} \times 1 / 2), 2.69$ (s, $1 \mathrm{H} \times 1 / 2), 3.89$ (d, $J=5.2 \mathrm{~Hz}, 1 \mathrm{H}), 4.03(\mathrm{~d}, J=5.2 \mathrm{~Hz}, 1 \mathrm{H}), 7.27-7.37(\mathrm{~m}, 5 \mathrm{H}) ;{ }^{13} \mathrm{C} \mathrm{NMR}\left(400 \mathrm{MHz}, \mathrm{CDCl}_{3}\right) \delta 15.0$, 17.9, 29.7, 36.1, 39.8, 40.3, 57.9, 69.2, 69.4, 127.4, 128.2, 128.3, 130.1, 130.2, 133.7; IR $\left(\mathrm{CHCl}_{3}\right)$ $3421,3018,1215,756 \mathrm{~cm}^{-1}$; HRMS (EI) calcd for $\mathrm{C}_{11} \mathrm{H}_{12}{ }^{79} \mathrm{BrClO}\left(\mathrm{M}^{+}\right)$273.9760, found 273.9757 .

\section{1,1-Dibromo-2-phenylcyclopropane (1p) ${ }^{12}$}

\section{1,1-Dibromo-2-butylcyclopropane $(1 \mathrm{q})^{13}$}

12) (a) Glayden, J.; Watson, D. W.; Chambers, M. Tetrahedron, 2005, 61, 3195. (b) Fleming, I.; Solay, M.; Stolwijk, F. J. Organomet. Chem. 1996, 521,121 .

13) (a) Nizovtsev, A. V.; Baird, M. S.; Bolesov, I. G. Tetrahedron, 2004, 60, 3717. (b) de Lang, R. J.; Brandsma, L. Synth. Commun.1998, $28,225$.

\section{$\left(1 R^{*}, 2 R^{*}\right)$-1-Chloro-2-phenylcyclopropanecarbaldehyde $(2 a)^{14}$ $\left(1 R^{*}, 2 S^{*}\right)$-1-Chloro-2-phenylcyclopropanecarbaldehyde (3a)}
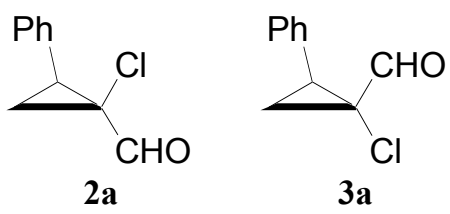

(Method I) A typical procedure: 1,1-Dichloro-2-phenylcyclopropane was stirred under $\mathrm{CO}$ pressure $(80 \mathrm{~atm})$ at $80{ }^{\circ} \mathrm{C}$ for $3 \mathrm{~h}$. AIBN $(33 \mathrm{mg}, 0.20 \mathrm{mmol})$ was added to the reaction mixture after temporary release of $\mathrm{CO}$ pressure. Then, the reaction mixture was stirred under $\mathrm{CO}$ pressure $(80 \mathrm{~atm})$ at $80{ }^{\circ} \mathrm{C}$ for $3 \mathrm{~h}$. After evacuation of excess $\mathrm{CO}$ at $\mathrm{rt}$, benzene was removed under reduced pressure. The residue was stirred for $1 \mathrm{~h}$ with $\mathrm{Et}_{2} \mathrm{O}(10 \mathrm{ml})$ and aqueous sat. $\mathrm{KF}$ solution $(10 \mathrm{ml})$. After Celite filtration, the separated organic phase was washed with water, brine, dried $\left(\mathrm{Na}_{2} \mathrm{SO}_{4}\right)$, and concentrated. The obtained crude product was purified by $\mathrm{SiO}_{2}$-column chromatography (hexane) to give an inseparable mixture of diastereoisomers $\mathbf{2} \mathbf{a}$ and $\mathbf{3 a}(\mathbf{2} \mathbf{a} / \mathbf{3} \mathbf{a}=3 / 1$, $92 \mathrm{mg}, 51 \%$ ). (Method II) A typical procedure: Following the procedure of method I, the reaction of 1-Bromo-1-chloro-2-phenylcyclopropane (1j) (230 mg, $1.00 \mathrm{mmol})$ with 0.2 equiv of AIBN (33 $\mathrm{mg}, 0.20 \mathrm{mmol}$, added once) in the place of 0.4 equiv of that ( 0.2 equiv added twice in method $\mathrm{I})$ gave an inseparable mixture of diastereoisomers $\mathbf{2 a}$ and 3a $(\mathbf{2 a} / \mathbf{3 a}=3 / 1,108 \mathrm{mg}, 60 \%)$. Relative configuration of minor product 3a was determined as cis-adduct based on known spectra data of 
trans-adduct $2 \mathbf{a}^{14}$. Additionally, due to the shielding effect ${ }^{15}$ (up field shift) of phenyl group, the chemical shift (CHO $8.88 \mathrm{ppm}$ ) of aldehyde 3a was assigned to that of cis-adduct. Ratio was analyzed by ${ }^{1} \mathrm{H}$ NMR. 2a and 3a: colorless oil; ${ }^{1} \mathrm{H}$ NMR $\left(400 \mathrm{MHz}, \mathrm{CDCl}_{3}\right) \delta 1.88(\mathrm{dd}, J=6.3 \mathrm{~Hz}$, $J=8.6 \mathrm{~Hz}, 1 \mathrm{H} \times 3 / 4), 2.00(\mathrm{dd}, J=6.8 \mathrm{~Hz}, J=9.9 \mathrm{~Hz}, 1 \mathrm{H} \mathrm{x} \mathrm{1/4),} 2.18(\mathrm{dd}, J=6.3 \mathrm{~Hz}, J=10.4 \mathrm{~Hz}$, $1 \mathrm{H} \mathrm{x} \mathrm{3/4),} 2.38(\mathrm{dd}, J=6.8 \mathrm{~Hz}, J=8.6 \mathrm{~Hz}, 1 \mathrm{H} \mathrm{x} \mathrm{1/4),} 3.00(\mathrm{dd}, J=8.6 \mathrm{~Hz}, J=10.4 \mathrm{~Hz}, 1 \mathrm{H} \mathrm{x} 3 / 4)$, $3.25(\mathrm{dd}, J=8.6 \mathrm{~Hz}, J=10.4 \mathrm{~Hz}, 1 \mathrm{H} \mathrm{x} \mathrm{1/4),} \mathrm{7.21-7.38} \mathrm{(m,} \mathrm{5H),} 8.88$ (s, $1 \mathrm{H} \mathrm{x} \mathrm{1/4),} 9.61$ (s, $1 \mathrm{H} \mathrm{x}$ 3/4); ${ }^{13} \mathrm{C}$ NMR $\left(100 \mathrm{MHz}, \mathrm{CDCl}_{3}\right) \delta 22.1,23.4,34.9,38.4,52.9,53.1,127.8,128.3,128.6,128.7$, 129.2, 129.4, 133.2, 133.5, 193.8, 197.1; IR (neat) 2843, 1716, $1497 \mathrm{~cm}^{-1}$; HRMS (EI) calcd for $\mathrm{C}_{10} \mathrm{H}_{9} \mathrm{ClO}\left(\mathrm{M}^{+}\right)$180.0342, found 180.0334 .

14) Verniest, G.; Bombeke, F.; Kulinkovich, O. G.; Kimpe, N. D. Tetrahedron Lett. 2002, 43, 599.

15) (a) Gaudner, A. Stereochemistry; Kagan, H. B., Ed.; Georg Thieme Verlag: Stuttgart, 1977, 1, 77. (b) Harada, T.; Katsuhira, T.; Hattori, K.; Oku, A. J. Org. Chem. 1993, 58, 2958.

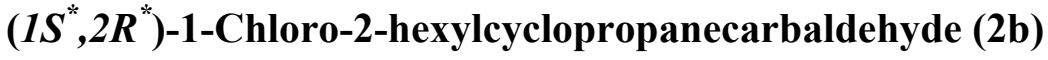

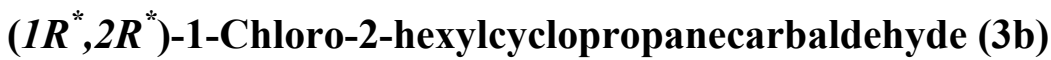

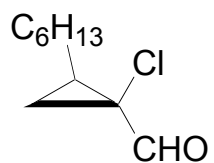

$\mathbf{2 b}$

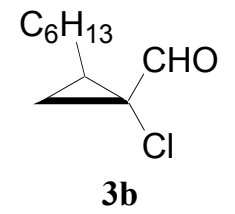

Following the procedure of method I, the reaction of $\mathbf{1 b}(181 \mathrm{mg}, 1.00$ mmol) gave the products $\mathbf{2 b}$ and $\mathbf{3 b}(\mathbf{2 b} / \mathbf{3 b}=3 / 1,84 \mathrm{mg}, 48 \%)$. Following the procedure of method II, the reaction of $1 \mathbf{k}(232 \mathrm{mg}, 1.00$ $\mathrm{mmol})$ gave the separable products $\mathbf{2} \mathbf{b}(110 \mathrm{mg}, 47 \%)$ and $\mathbf{3 b}(36 \mathrm{mg}$, 16\%). Purification was carried out by $\mathrm{SiO}_{2}$-column chromatography (hexane/AcOEt = 30/1). Based on the the deshielding effect of alkyl group, the chemical shift (CHO $9.56 \mathrm{ppm}$, down field shift) of aldehyde $\mathbf{3 b}$ was assigned to that of cis-adduct. $\mathbf{2 b}$ : colorless oil; ${ }^{1} \mathrm{H}$ NMR $\left(400 \mathrm{MHz}, \mathrm{CDCl}_{3}\right)$ $\delta 0.89(\mathrm{t}, J=7.1 \mathrm{~Hz}, 3 \mathrm{H}), 1.13-1.16(\mathrm{~m}, 1 \mathrm{H}), 1.23-1.37$ (m, 7H), 1.41-1.50 (m, 2H), 1.58-1.68 (m, $3 \mathrm{H}), 1.79-1.82(\mathrm{~m}, 1 \mathrm{H}), 9.39(\mathrm{~s}, 1 \mathrm{H}) ;{ }^{13} \mathrm{C} \mathrm{NMR}\left(100 \mathrm{MHz}, \mathrm{CDCl}_{3}\right) \delta$ 14.0, 22.5, 25.3, 27.1, 28.7, 29.3, 31.6, 37.1, 50.0, 196.5; IR (neat) 2923, 2855, $1717 \mathrm{~cm}^{-1}$; HRMS (EI) calcd for $\mathrm{C}_{10} \mathrm{H}_{17} \mathrm{ClO}\left(\mathrm{M}^{+}\right)$ 188.0968, found 188.0977. 3b: colorless oil; ${ }^{1} \mathrm{H} \mathrm{NMR}\left(400 \mathrm{MHz}, \mathrm{CDCl}_{3}\right) \delta 0.89(\mathrm{t}, J=7.1 \mathrm{~Hz}, 3 \mathrm{H})$, 1.24-1.43 (m, 8H), 1.47-1.57 (m, 2H), 1.60-1.68 (m, 2H), 1.85-1.93 (m, 1H), $9.56(\mathrm{~s}, 1 \mathrm{H}) ;{ }^{13} \mathrm{C}$ NMR $\left(100 \mathrm{MHz}, \mathrm{CDCl}_{3}\right) \delta$ 14.0, 22.5, 25.3, 28.7, 28.9, 29.1, 29.8, 31.6, 52.7, 197.4; IR (neat) 2856, 1697, 1440, $1290 \mathrm{~cm}^{-1}$; HRMS (EI) calcd for $\mathrm{C}_{10} \mathrm{H}_{17} \mathrm{ClO}\left(\mathrm{M}^{+}\right)$188.0968, found 188.0972.

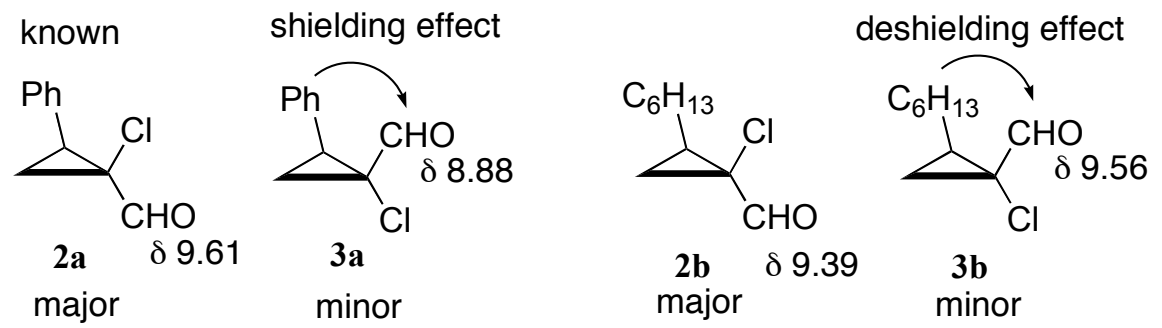

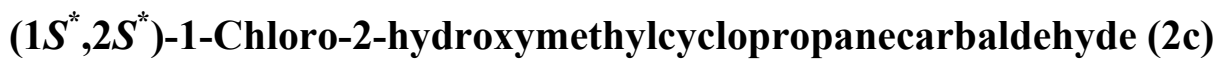

$\left(1 R^{*}, 2 R^{*}, 5 S^{*}\right)$ - and $\left(1 R^{*}, 2 S^{*}, 5 S^{*}\right)-1-$ Chloro-3-oxabicyclo[3.1.0]hexan-2-ol (3c) 


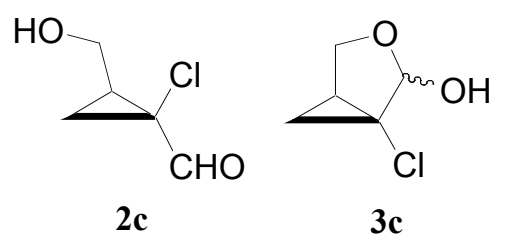

Following the procedure of method I, the reaction of 1c $(141 \mathrm{mg}$, $1.00 \mathrm{mmol}$ ) gave the products $\mathbf{2 c}(14 \mathrm{mg}, 10 \%)$ and $3 \mathbf{c}$ (a couple of diastereoisomeric lactols, inseparable mixture, ratio $=5 / 1$ in $\mathrm{CDCl}_{3}$, $56 \mathrm{mg}, 38 \%$ ). Aldehyde 2c and lactol 3c was separated by $\mathrm{SiO}_{2}$ column chromatography (hexane/AcOEt $=5 / 1)$. 2c: colorless oil; ${ }^{1} \mathrm{H}$ NMR $\left(400 \mathrm{MHz}, \mathrm{CDCl}_{3}\right) \delta$ $1.36(\mathrm{dd}, J=5.8,8.1 \mathrm{~Hz}, 1 \mathrm{H}),(\mathrm{m}, 1 \mathrm{H}), 1.86(\mathrm{dd}, J=5.8 \mathrm{~Hz}, J=10.1 \mathrm{~Hz}, 1 \mathrm{H}), 2.02-2.10(\mathrm{~m}, 1 \mathrm{H})$ $3.75(\mathrm{dd}, J=8.8 \mathrm{~Hz}, J=12.0 \mathrm{~Hz}, 1 \mathrm{H}), 4.06(\mathrm{dd}, J=4.4,12.0 \mathrm{~Hz}, 1 \mathrm{H}), 9.42(\mathrm{~s}, 1 \mathrm{H}) ;{ }^{13} \mathrm{C}$ NMR $(100$ $\left.\mathrm{MHz}, \mathrm{CDCl}_{3}\right) \delta 22.7,30.7,51.4,61.7,196.4$; IR (neat) 3487, 2954, 2863, $1725 \mathrm{~cm}^{-1}$; HRMS (EI) calcd for $\mathrm{C}_{5} \mathrm{H}_{7} \mathrm{ClO}_{2}\left(\mathrm{M}^{+}\right)$134.0135, found 134.0130. 3c: colorless oil; ${ }^{1} \mathrm{H} \mathrm{NMR}\left(400 \mathrm{MHz}, \mathrm{CDCl}_{3}\right)$ $\delta 0.99(\mathrm{dd}, J=4.0 \mathrm{~Hz}, J=6.0 \mathrm{~Hz}, 1 \mathrm{H}), 1.23(\mathrm{dd}, J=6.0 \mathrm{~Hz}, J=8.4 \mathrm{~Hz}, 1 \mathrm{H}), 1.96-1.99(\mathrm{~m}, 1 \mathrm{H})$, $3.47(\mathrm{dd}, J=7.1 \mathrm{~Hz}, J=11.6 \mathrm{~Hz}, 1 \mathrm{H}), 3.59(\mathrm{dd}, J=7.1 \mathrm{~Hz}, J=11.6 \mathrm{~Hz}, 1 \mathrm{H}), 5.27(\mathrm{~d}, J=4.0 \mathrm{~Hz}$, $1 \mathrm{H} x$ 5/6), 5.43 (d, $J=7.1 \mathrm{~Hz}, 1 \mathrm{H} \times 1 / 6) ;{ }^{13} \mathrm{C} \mathrm{NMR}\left(100 \mathrm{MHz}, \mathrm{CDCl}_{3}\right) \delta$ 15.0, 22.4, 62.8, 67.7, 98.6; IR (neat) 3489, 2944, $2867 \mathrm{~cm}^{-1}$; HRMS (EI) calcd for $\mathrm{C}_{5} \mathrm{H}_{7} \mathrm{ClO}_{2}\left(\mathrm{M}^{+}\right)$134.0135, found 134.0139.

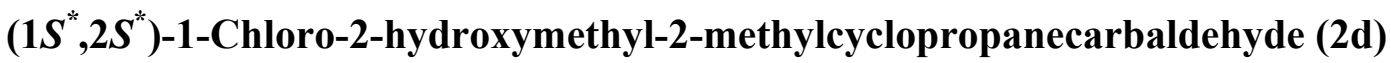 $\left(1 R^{*}, 2 R^{*}, 5 S^{*}\right)$ - and $\left(1 R^{*}, 2 S^{*}, 5 S^{*}\right)-1-C h l o r o-5-m e t h y l-3-0 x a b i c y c l o[3.1 .0]$ hexan-2-ol (3d)}

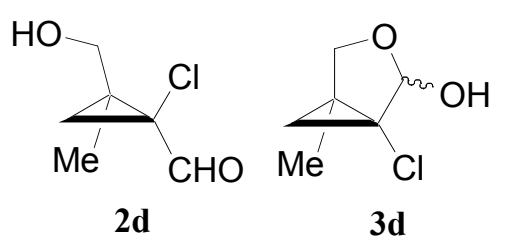

Following the procedure of method I, the reaction of $\mathbf{1 d}(155 \mathrm{mg}$, $1.00 \mathrm{mmol}$ ) gave the products $\mathbf{2 d}(15 \mathrm{mg}, 15 \%)$ and $\mathbf{3 d}$ (a couple of diastereoisomeric lactols, ratio $=1 / 4,74 \mathrm{mg}, 45 \%$ ). Following the procedure of method II, the reaction of $1 \mathrm{n}(199 \mathrm{mg}, 1.00 \mathrm{mmol})$ gave the products $2 \mathbf{d}(18 \mathrm{mg}, 16 \%)$ and $\mathbf{3 d}$ (a couple of diastereoisomeric lactols, ratio $=4 / 1$ in $\mathrm{CDCl}_{3}, 74 \mathrm{mg}, 46 \%$ ). Aldehyde $\mathbf{2 d}$ and lactol 3d was separated by $\mathrm{SiO}_{2}-$ column chromatography (hexane/AcOEt $=5 / 1)$. 2 d: colorless oil; ${ }^{1} \mathrm{H} \mathrm{NMR}\left(400 \mathrm{MHz}, \mathrm{CDCl}_{3}\right) \delta$ $1.34(\mathrm{~s}, 3 \mathrm{H}), 1.42$ (d, $J=6.3 \mathrm{~Hz}, 1 \mathrm{H}), 1.83(\mathrm{~d}, J=6.3 \mathrm{~Hz}, 1 \mathrm{H}), 3.77$ (d, $J=12.1 \mathrm{~Hz}, 1 \mathrm{H}), 3.90(\mathrm{~d}, J$ $=12.1 \mathrm{~Hz}, 1 \mathrm{H}), 9.60(\mathrm{~s}, 1 \mathrm{H}) ;{ }^{13} \mathrm{C} \mathrm{NMR}\left(100 \mathrm{MHz} \mathrm{CDCl}_{3}\right) \delta 15.0,27.8,36.7,55.9,68.0,195.9$; IR (neat) 3410, 2936, 2874, $1717 \mathrm{~cm}^{-1}$; $\operatorname{HRMS}$ (EI) calcd for $\mathrm{C}_{6} \mathrm{H}_{9} \mathrm{ClO}_{2}\left(\mathrm{M}^{+}\right)$148.0291, found 148.0289. 3d: colorless oil; ${ }^{1} \mathrm{H}-\mathrm{NMR}\left(400 \mathrm{MHz}, \mathrm{CDCl}_{3}\right) \delta 0.83(\mathrm{~d}, J=5.8 \mathrm{~Hz}, 1 \mathrm{H} \mathrm{x} 1 / 5), 0.91$ (d, $J$

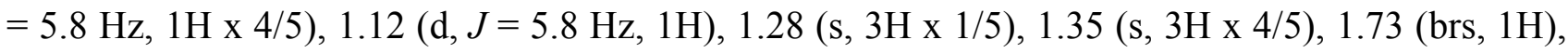
$2.97(\mathrm{dd}, J=4.0 \mathrm{~Hz}, J=7.3 \mathrm{~Hz}, 1 \mathrm{H}), 3.72(\mathrm{~d}, J=8.4 \mathrm{~Hz}, 1 \mathrm{H}), 3.86(\mathrm{~d}, J=8.4 \mathrm{~Hz}, 1 \mathrm{H} \mathrm{x} 1 / 5, \mathrm{OH})$, $3.94(\mathrm{~d}, J=8.4 \mathrm{~Hz}, 1 \mathrm{H} 4 / 5, \mathrm{OH}), 5.32\left(\mathrm{~d}, J=4.0 \mathrm{~Hz}, 1 \mathrm{H}\right.$ x 4/5), $5.38(\mathrm{~d}, J=7.1 \mathrm{~Hz}, 1 \mathrm{H} \mathrm{x} 1 / 5) ;{ }^{13} \mathrm{C}$ NMR (100 MHz, $\left.\mathrm{CDCl}_{3}\right) \delta 13.3,22.6,27.1,52.0,71.5,98.3$; IR (neat) 3462, 2842, $1476 \mathrm{~cm}^{-1}$; HRMS (EI) calcd for $\mathrm{C}_{6} \mathrm{H}_{9} \mathrm{ClO}_{2}\left(\mathrm{M}^{+}\right)$148.0291, found 148.0298 .
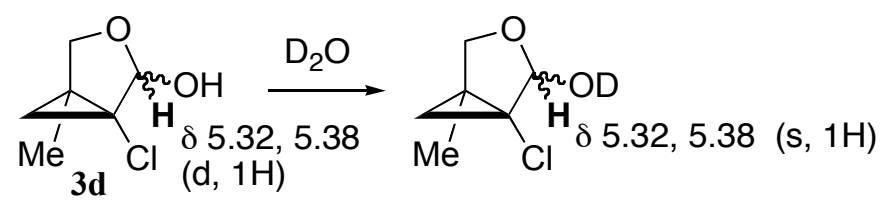

D-H exchange of lactol 3d using $\mathrm{D}_{2} \mathrm{O}$ gave deuterated lactol. The spectrum of $\mathrm{OH}$ disappeared and coupling pattern of the anomeric proton $[5.32(\mathrm{~d}, J=4.0 \mathrm{~Hz}, 1 \mathrm{H} \mathrm{x}$

4/5), $5.38(\mathrm{~d}, J=7.1 \mathrm{~Hz}, 1 \mathrm{H} \times 1 / 5)]$ changed into singlet. 


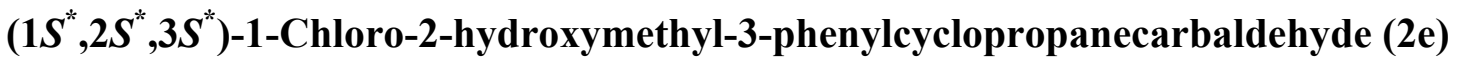

$\left(1 R^{*}, 2 R^{*}, 5 S^{*}, 6 S^{*}\right)$ - and $\left(1 R^{*}, 2 S^{*}, 5 S^{*}, 6 S^{*}\right)-(1-C h l o r o-6-p h e n y l-3-o x a b i c y c l o[3.1 .0]$ hexan-2-ol (3e)

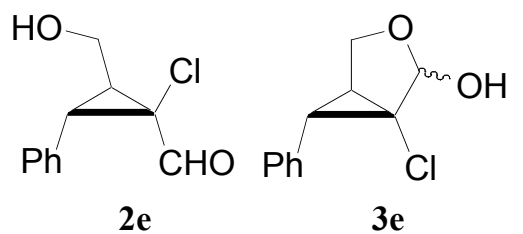

Following the procedure of method I, the reaction of $1 \mathrm{e}(232 \mathrm{mg}$, $1.00 \mathrm{mmol})$ gave the products $2 \mathbf{e}(20 \mathrm{mg}, 9 \%)$ and $\mathbf{3 e}$ (a couple of diastereoisomeric lactols, ratio $=5 / 1$ in $\mathrm{CDCl}_{3}, 81 \mathrm{mg}, 37 \%$ ). Aldehyde $\mathbf{2}$ e and lactol $\mathbf{3} \mathbf{e}$ separated by $\mathrm{SiO}_{2}$-column chromatography (hexane/AcOEt $=4 / 1)$. 2e: colorless oil; ${ }^{1} \mathrm{H}$ NMR $\left(400 \mathrm{MHz}, \mathrm{CDCl}_{3}\right) \delta 1.97(\mathrm{brs}$, $1 \mathrm{H}), 2.81(\mathrm{dt}, J=5.3,8.6 \mathrm{~Hz}, 1 \mathrm{H}), 3.08(\mathrm{~d}, J=8.6 \mathrm{~Hz}, 1 \mathrm{H}), 3.96(\mathrm{dd}, J=8.6 \mathrm{~Hz}, J=12.1 \mathrm{~Hz}, 1 \mathrm{H})$, $4.20(\mathrm{dd}, J=5.3 \mathrm{~Hz}, J=12.1 \mathrm{~Hz}, 1 \mathrm{H}), 7.20-7.26(\mathrm{~m}, 1 \mathrm{H}),(\mathrm{m}, 1 \mathrm{H}), 7.28-7.34(\mathrm{~m}, 4 \mathrm{H}), 8.95(\mathrm{~s}, 1 \mathrm{H})$;

${ }^{13} \mathrm{C} \mathrm{NMR}\left(100 \mathrm{MHz}, \mathrm{CDCl}_{3}\right) \delta$ 32.0, 33.3, 55.6, 62.0, 128.4, 129.0, 129.1, 133.1, 193.8; IR (neat) 3402, 2873, 1717, $1497 \mathrm{~cm}^{-1}$; HRMS (EI) calcd for $\mathrm{C}_{11} \mathrm{H}_{11} \mathrm{ClO}_{2}\left(\mathrm{M}^{+}\right)$210.0448, found 210.0483 . 3e: colorless crystals; mp 79-82 ${ }^{\circ} \mathrm{C} ;{ }^{1} \mathrm{H}$ NMR $\left(400 \mathrm{MHz}, \mathrm{CDCl}_{3}\right) \delta 1.78-1.85(\mathrm{~m}, 1 \mathrm{H}), 2.28(\mathrm{~d}, J=$ $4.8 \mathrm{~Hz}, 1 \mathrm{H}$ x 5/6), 2.65 (d, $J=4.8 \mathrm{~Hz}, 1 \mathrm{H} \mathrm{x} \mathrm{1/6),} 3.83$ (d, $J=8.6 \mathrm{~Hz}, 1 \mathrm{H}$ x 5/6), 3.96 (d, $J=9.1 \mathrm{~Hz}$, $1 \mathrm{H} \mathrm{x} \mathrm{1/6),} 4.00(\mathrm{dd}, J=3.0 \mathrm{~Hz}, J=9.1 \mathrm{~Hz}, 1 \mathrm{H} \times 1 / 6), 4.28(\mathrm{dd}, J=2.5 \mathrm{~Hz}, J=8.6 \mathrm{~Hz}, 1 \mathrm{H} \times 5 / 6)$, $5.34(\mathrm{~s}, 1 \mathrm{H} \times 5 / 6), 5.54(\mathrm{~s}, 1 \mathrm{H} \times 1 / 6), 7.14-7.20(\mathrm{~m}, 3 \mathrm{H}), 7.26-7.29$ (m, 2H); ${ }^{13} \mathrm{C} \mathrm{NMR}(100 \mathrm{MHz}$, $\left.\mathrm{CDCl}_{3}\right) \delta 30.5,37.5,54.3,67.5,98.1,127.1,128.1,128.8,134.6 ; \mathrm{IR}\left(\mathrm{CHCl}_{3}\right) 3453,2876,1489 \mathrm{~cm}^{-1}$; HRMS (EI) calcd for $\mathrm{C}_{11} \mathrm{H}_{11} \mathrm{ClO}_{2}\left(\mathrm{M}^{+}\right) 210.0448$, found 210.0445 .

$\left(1 R^{*}, 2 R^{*}, 5 S^{*}, 6 R^{*}\right)$ - and $\left(1 R^{*}, 2 S^{*}, 5 S^{*}, 6 R^{*}\right)-1-C h l o r o-5-m e t h y l-6-p h e n y l-3-0 x a b i c y c l o[3.1 .0]$ hexan-2-ol (3f)

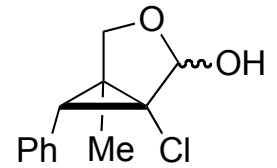

3f

Following the procedure of method I, the reaction of $1 \mathbf{f}(231 \mathrm{mg}, 1.00 \mathrm{mmol})$ gave the lactol $3 f$ ( $94 \mathrm{mg}, 42 \%)$. Following the procedure of method II, the reaction of $10(100 \mathrm{mg}, 0.36 \mathrm{mmol})$ gave the lactol $3 \mathrm{f}(34 \mathrm{mg}, 41 \%)$. A couple of diastereoisomeric lactols apparently existed (ratio $=4 / 1$ in $\mathrm{CDCl}_{3}$ ). $\mathbf{3 f}$ : colorless crystals; mp 88-91 ${ }^{\circ} \mathrm{C} ;{ }^{1} \mathrm{H}$ NMR (400 MHz, $\left.\mathrm{CDCl}_{3}\right) \delta 1.18$ (s, 3H x 1/5), 1.25 (s, 3H x 4/5), 2.36 (s, $1 \mathrm{H} \times 4 / 5), 2.63(\mathrm{~s}, 1 \mathrm{H} \times 1 / 5), 3.85(\mathrm{~d}, J=8.3 \mathrm{~Hz}, 1 \mathrm{H} \times 1 / 5), 3.96(\mathrm{~d}, J=8.3 \mathrm{~Hz}, 1 \mathrm{H} \times \mathrm{4} / 5), 4.07(\mathrm{~d}, J$ $=8.3 \mathrm{~Hz}, 1 \mathrm{H}), 5.49(\mathrm{~s}, 1 \mathrm{H} \times 4 / 5), 5.53(\mathrm{~s}, 1 \mathrm{H} \times 1 / 5), 7.28-7.35(\mathrm{~m}, 5 \mathrm{H}) ;{ }^{13} \mathrm{C} \mathrm{NMR}(400 \mathrm{MHz}$, $\left.\mathrm{CDCl}_{3}\right) \delta 10.6,29.5,31.0,56.2,72.9,99.0,126.8,128.1,130.7,133.8 ; \mathrm{IR}\left(\mathrm{CHCl}_{3}\right) 3387,1501 \mathrm{~cm}^{-1}$; HRMS (EI) calcd for $\mathrm{C}_{12} \mathrm{H}_{13} \mathrm{ClO}_{2}\left(\mathrm{M}^{+}\right)$224.0604, found 224.0595 .

\section{endo-7-Chlorobicyclo[4.1.0]heptane-7-carbaldehyde (2g) (trans-adduct)}

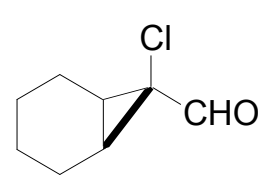

$2 \mathrm{~g}$

Following the procedure of method I, the reaction of $1 \mathrm{~g}(165 \mathrm{mg}, 1.00 \mathrm{mmol})$ gave the products $2 \mathrm{~g}(87 \mathrm{mg}, 55 \%)$. Following the procedure of method II, The reaction of 11 (210 $\mathrm{mg}, 1.00 \mathrm{mmol})$ gave the product $2 \mathrm{~g}(116 \mathrm{mg}, 73 \%)$. Based on our previous report ${ }^{16}$, the relative configuration was determined by a NOESY measurement of $\mathbf{2 g - 2}$, which was derived from $\mathbf{2 g}$. The stereochemical assignment is also possible by a comparison of chemical shift (CHO) of $\mathbf{2 g}$ with that of $\mathbf{1 4 l}$. Due to a deshielding effect of alkyl substituent, chemical shift of aldehyde-proton of cis-adduct appears at lower field. The chemical 
shift (CHO $9.72 \mathrm{ppm}$ ) of aldehyde $\mathbf{1 4 l}$ was assigned to that of cis-adduct, hence, that (CHO 9.43 $\mathrm{ppm}$ ) of $\mathbf{2 g}$ was assigned to that of trans-adduct.

2g: colorless oil; ${ }^{1} \mathrm{H}$ NMR $\left(400 \mathrm{MHz}, \mathrm{CDCl}_{3}\right) \delta$ 1.24-1.35 (m, 2H), 1.39-1.47 (m, 2H), 1.62-1.69 (m, 2H), 1.90-1.94 (m, 2H), 1.97-2.06 (m, 2H), 9.43 (s, 1H, CHO); ${ }^{13} \mathrm{C}$ NMR (100 MHz, $\left.\mathrm{CDCl}_{3}\right) \delta 18.7$, 20.8, 25.9, 58.7, 198.5; IR (neat) 2939, 2858, 1709, $1447 \mathrm{~cm}^{-1}$; HRMS (EI) calcd for $\mathrm{C}_{8} \mathrm{H}_{11} \mathrm{ClO}\left(\mathrm{M}^{+}\right)$ 158.0498, found 158.0498 .
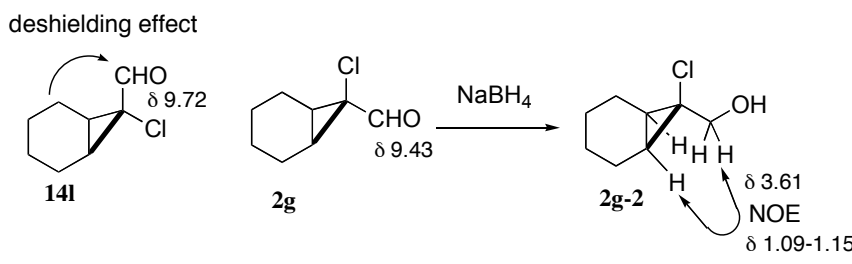

2g-2: ${ }^{1} \mathrm{H}$ NMR $\left(400 \mathrm{MHz}, \mathrm{CDCl}_{3}\right) \delta \underline{1.09-1.15}$ (m, 2H), 1.19-1.28 (m, 2H), 1.32-1.41 (m, 2H), 1.57-1.65 (m, 2H), 1.90-1.99 (m, 2H), $\underline{3.61}(\mathrm{~d}$,

16) Tanabe, Y.; Wakimura, K.; Nishii Y. Tetrahedron Lett. 1996, 37, 1837

endo-9-Chlorobicyclo[6.1.0]nonane-9-carbaldehyde (2h) (trans-adduct)

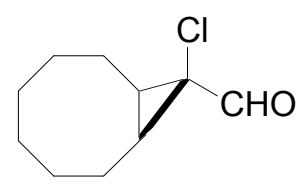

$2 \mathbf{h}$ Following the procedure of method I, the reaction of $\mathbf{1} \mathbf{h}(193 \mathrm{mg}, 1.00 \mathrm{mmol})$ gave the products $\mathbf{2 h}(88 \mathrm{mg}, 47 \%)$. Following the procedure of method II, the reaction of $1 \mathbf{m}(238 \mathrm{mg}, 0.36 \mathrm{mmol})$ gave the product $\mathbf{2 h}(125 \mathrm{mg}, 67 \%)$. Based on the analogy of spectra data of $\mathbf{2 g}$, the relative configuration of $\mathbf{2} \mathbf{h}$ was determined as trans-adduct. $2 \mathbf{h}$ : colorless oil; ${ }^{1} \mathrm{H}$ NMR $\left(400 \mathrm{MHz}, \mathrm{CDCl}_{3}\right) \delta 1.39-1.52,(\mathrm{~m}, 6 \mathrm{H})$, 1.59-1.74 (m, 6H), 1.82-1.93 (m, 2H), 9.43 (s, 1H); ${ }^{13} \mathrm{C}$ NMR (100 MHz, $\left.\mathrm{CDCl}_{3}\right) \delta$ 22.4, 26.3, 28.1, 31.9, 57.4, 198.5; IR (neat) 2923, 2854, $1713 \mathrm{~cm}^{-1}$; HRMS (EI) calcd for $\mathrm{C}_{10} \mathrm{H}_{15} \mathrm{ClO}\left(\mathrm{M}^{+}\right)$186.0811, found 186.0814 .

endo-7-Bromobicyclo[4.1.0]heptane-7-carbaldehyde (2i) (trans-adduct)

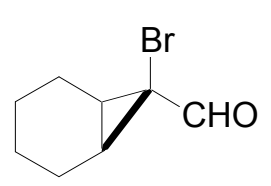

$2 \mathbf{i}$

Following the procedure of method II, the reaction of $1 \mathbf{i}(253 \mathrm{mg}, 1.00 \mathrm{mmol})$ gave the product $2 \mathbf{i}(28 \mathrm{mg}, 14 \%) .2 \mathbf{i}$ : colorless oil; ${ }^{1} \mathrm{H}$ NMR $\left(400 \mathrm{MHz}, \mathrm{CDCl}_{3}\right) \delta 1.18$ $1.27(\mathrm{~m}, 2 \mathrm{H}), 1.31-1.40(\mathrm{~m}, 2 \mathrm{H}), 1.54-1.61$ (m, 2H), 1.80-1.86 (m, 2H), 1.89-1.98 $(\mathrm{m}, 2 \mathrm{H}), 9.36(\mathrm{~s}, 1 \mathrm{H}) ;{ }^{13} \mathrm{C} \mathrm{NMR}\left(100 \mathrm{MHz}, \mathrm{CDCl}_{3}\right) \delta$ 18.8, 21.0, 32.9, 44.2, 195.8;

IR (neat) 2939, 2858, 1709, 1447; HRMS (EI) calcd for $\mathrm{C}_{8} \mathrm{H}_{11} \mathrm{BrO}\left(\mathrm{M}^{+}\right)$201.9993, found 201.9997.

The relative configuration of aldehyde $\mathbf{2} \mathbf{i}$ was determined as trans-adduct by the comparison of chemical shift (CHO) of $\mathbf{1 4} \mathbf{i}$ (another stereoisomer) with that of $\mathbf{2 i}$. Based on a deshielding effect of the alkyl substituent, the chemical shift (CHO: $9.55 \mathrm{ppm}$ ) of aldehyde 14i was assigned to that of cis-adduct, hence, that (CHO: $9.36 \mathrm{ppm}$ ) of $\mathbf{2} \mathbf{i}$ was assigned to that of trans-adduct.

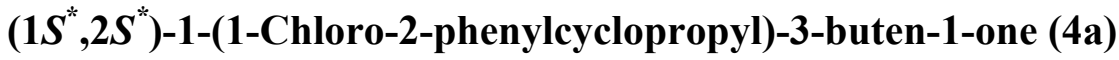

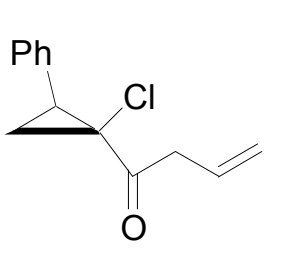

1,1-Dichloro-2-phenylcyclopropane (1a) (187 mg, $1.00 \mathrm{mmol})$, AIBN (33 mg, $0.20 \mathrm{mmol}), \mathrm{Bu}_{3} \mathrm{Sn}\left(\mathrm{CH}_{2} \mathrm{CH}=\mathrm{CH}_{2}\right)(662 \mathrm{mg}, 2.00 \mathrm{mmol})$, and benzene $(30 \mathrm{~mL})$ 
were placed in a $100-\mathrm{mL}$ stainless steel autoclave with a insert glass tube. The reaction mixture was stirred under $\mathrm{CO}$ pressure $(40 \mathrm{~atm})$ at $80{ }^{\circ} \mathrm{C}$ for $12 \mathrm{~h}$. AIBN (33 mg, $\left.0.20 \mathrm{mmol}\right)$ was added to the reaction mixture after temporary release of $\mathrm{CO}$ pressure. Then, the reaction mixture was stirred under $\mathrm{CO}$ pressure $(40 \mathrm{~atm})$ at $80{ }^{\circ} \mathrm{C}$ for $12 \mathrm{~h}$. After evacuation of excess $\mathrm{CO}$ at room temperature, benzene was removed under reduced pressure. The residue was stirred for $1 \mathrm{~h}$ with $\mathrm{Et}_{2} \mathrm{O}(10 \mathrm{ml})$ and aqueous sat. KF solution $(10 \mathrm{ml})$. The residue was stirred for $1 \mathrm{~h}$ with $\mathrm{Et}_{2} \mathrm{O}(10 \mathrm{ml})$ and aqueous sat. KF solution $(10 \mathrm{ml})$. After Celite filtration, the separated organic phase was washed with water, brine, dried $\left(\mathrm{Na}_{2} \mathrm{SO}_{4}\right)$, and concentrated. The obtained crude product was purified by $\mathrm{SiO}_{2}$-column chromatography (hexane/EtOAc $=3 / 1)$ to give the desired product $4 \mathbf{a}(113 \mathrm{mg}, 51 \%)$. (b) (Method IV). Following the procedure of method III, the reaction of 1-Bromo-1-chloro-2phenylcyclopropane (1j) $(210 \mathrm{mg}, 1.00 \mathrm{mmol})$ with 0.2 equiv of AIBN (33 mg, $0.20 \mathrm{mmol}$, added once) in the place of 0.4 equiv of that ( 0.2 equiv added twice in method III) gave the desired product product 4a (126 mg, 58\%). 4a: colorless oil; ${ }^{1} \mathrm{H} \mathrm{NMR}\left(400 \mathrm{MHz}, \mathrm{CDCl}_{3}\right) \delta 1.73(\mathrm{dd}, J=7.0 \mathrm{~Hz}, J=$ $10.1 \mathrm{~Hz}, 1 \mathrm{H}), 2.21(\mathrm{dd}, J=7.0 \mathrm{~Hz}, J=10.1 \mathrm{~Hz} 1 \mathrm{H}), 3.01(\mathrm{dd}, J=7.0 \mathrm{~Hz}, J=10.1 \mathrm{~Hz}, 1 \mathrm{H}) 3.68(\mathrm{~m}$, 2H), 5.16-5.26 (m, 2H), 5.94-6.04 (m, 1H), 7.20-7.24 (m, 2H), 7.29-7.37 (m, 3H); ${ }^{13} \mathrm{C}$ NMR $(100$ $\left.\mathrm{MHz}, \mathrm{CDCl}_{3}\right) \delta 25.0,36.3,44.8,52.5,119.0,127.6,128.1,129.3,130.2,134.6,204.5$; IR (neat) 2843, 1716, 1640, $1497 \mathrm{~cm}^{-1}$; HRMS (EI) calcd for $\mathrm{C}_{13} \mathrm{H}_{13} \mathrm{ClO}\left(\mathrm{M}^{+}\right) 220.0655$, found 220.0656.

In order to determine the relative configuration, aldehyde 2a (trans-adduct) which was stereoselectively prepared by known method ${ }^{14}$ converted into ketone $\mathbf{4 a}$ in two steps [(1) $\left(\mathrm{CH}_{2}=\mathrm{CHCH}_{2}\right) \mathrm{MgBr}$, (2) Jones' oxidation]. Thus, the relative configuration of $\mathbf{4 a}$ was assigned as trans-adduct.
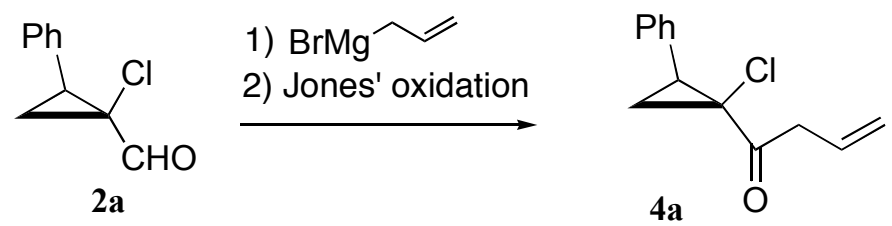

\section{$\left(1 S^{*}, 2 R^{*}\right)-1-(1-C h l o r o-2-p e n t y l c y c l o p r o p y l)-3-b u t e n-1-o n e ~(4 b)$}

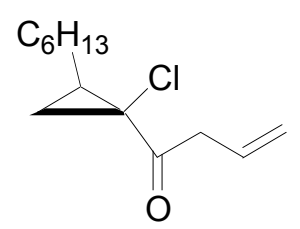

4b

Following the procedure of method III, the reaction of $\mathbf{1 b}(181 \mathrm{mg}, 1.00 \mathrm{mmol})$ gave the product $\mathbf{4 b}(108 \mathrm{mg}, 47 \%)$. Following the procedure of method IV, the reaction of $1 \mathbf{k}(232 \mathrm{mg}, 1.00 \mathrm{mmol})$ gave the product $\mathbf{4 b}(122 \mathrm{mg}, 53 \%)$. Based on the analogy of spectra data of $\mathbf{2} \mathbf{b}$, the relative configuration of $\mathbf{4 b}$ was determined as trans-adduct. 4b: colorless oil; ${ }^{1} \mathrm{H}$ NMR $\left(400 \mathrm{MHz}, \mathrm{CDCl}_{3}\right) \delta 0.89$ $(\mathrm{m}, 3 \mathrm{H}), 0.95-0.98(\mathrm{~m}, 1 \mathrm{H}), 1.23-1.36(\mathrm{~m}, 6 \mathrm{H}), 1.39-1.49(\mathrm{~m}, 2 \mathrm{H}), 1.53-1.69(\mathrm{~m}, 3 \mathrm{H}), 1.82-1.85(\mathrm{~m}$, $1 \mathrm{H}), 3.62-3.65(\mathrm{~m}, 2 \mathrm{H}), 5.13-5.21(\mathrm{~m}, 2 \mathrm{H}), 5.90-6.00(\mathrm{~m}, 1 \mathrm{H}) ;{ }^{13} \mathrm{C} \mathrm{NMR}\left(100 \mathrm{MHz}, \mathrm{CDCl}_{3}\right) \delta 14.0$, 22.6, 28.8, 29.0, 29.6, 31.7, 31.9, 44.7, 52.4, 118.7, 205.4; IR (neat) 2927, 1701, $1620 \mathrm{~cm}^{-1}$; HRMS (EI) calcd for $\mathrm{C}_{13} \mathrm{H}_{21} \mathrm{ClO}\left(\mathrm{M}^{+}\right) 228.1281$, found 228.1286 . 
Following the procedure of method III, the reaction of $\mathbf{1 d}(155 \mathrm{mg}, 1.00 \mathrm{mmol})$

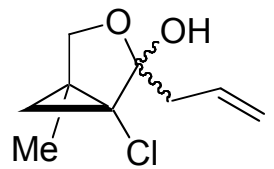

5d gave the products $\mathbf{5 d}(72 \mathrm{mg}, 38 \%)$. Following the procedure of method IV, the reaction of $1 \mathbf{n}(199 \mathrm{mg}, 1.00 \mathrm{mmol})$ gave the lactol $\mathbf{5 d}(129 \mathrm{mg}, 68 \%)$. A couple of diastereoisomeric lactols apparently existed (ratio $=2 / 1$ in $\mathrm{CDCl}_{3}$ ). Based on the analogy of spectra data of lactol $\mathbf{3 d}$, the relative configuration of $\mathbf{4 b}$ was tentatively determined as cis-adduct (lactol). 5d: colorless oil; ${ }^{1} \mathrm{H}$ NMR (400 MHz, $\left.\mathrm{CDCl}_{3}\right) \delta 0.87$ $(\mathrm{d}, J=5.8 \mathrm{~Hz}, 1 \mathrm{H} \times 1 / 3), 0.91(\mathrm{~d}, J=4.8 \mathrm{~Hz}, 1 \mathrm{H} \mathrm{x} \mathrm{2/3),} 1.01(\mathrm{~d}, J=4.8 \mathrm{~Hz}, 1 \mathrm{H} \mathrm{x} 2 / 3), 1.13(\mathrm{~d}, J=$ $5.8 \mathrm{~Hz}, 1 \mathrm{H} \times 1 / 3), 1.34$ (s, 3H), 1.55 (brs, $1 \mathrm{H} \times 2 / 3$ ), 1.60 (brs, $1 \mathrm{H} \times 1 / 3), 2.15-2.21$ (m, $1 \mathrm{H} \times 2 / 3$ ), 2.49-2.55 (m, $1 \mathrm{H} \times 1 / 3), 2.58-2.64(\mathrm{~m}, 1 \mathrm{H} \times 1 / 3), 2.66-2.72(\mathrm{~m}, 1 \mathrm{H} \times 1 / 3), 3.70(\mathrm{~d}, J=8.4 \mathrm{~Hz}, 1 \mathrm{H} \mathrm{x}$ 1/3), $3.88(\mathrm{~d}, J=8.4 \mathrm{~Hz}, 1 \mathrm{H} \times 1 / 3), 4.00(\mathrm{~d}, J=9.1 \mathrm{~Hz}, 1 \mathrm{H} \times 2 / 3), 4.19$ (d, $J=9.1 \mathrm{~Hz}, 1 \mathrm{H} \times 2 / 3)$, 5.04-5.07 (m, $1 \mathrm{H} \times 2 / 3), 5.09-5.10(\mathrm{~m}, 1 \mathrm{H} \times 2 / 3), 5.23-5.24(\mathrm{~m}, 1 \mathrm{H} \times 1 / 3), 5.26-5.29$ (m, $1 \mathrm{H} \times 1 / 3)$, 5.83-6.00 (m, 1H); ${ }^{13} \mathrm{C}$ NMR $\left(100 \mathrm{MHz}, \mathrm{CDCl}_{3}\right) \delta 13.5,14.6,22.7,23.0,27.5,28.2,30.4,40.0$, 54.4, 54.6, 71.2, 72.9, 103.9, 116.8, 120.1, 121.3, 131.7, 134.3; IR (neat) 3468, 1763, $1634 \mathrm{~cm}^{-1}$; HRMS (EI) calcd for $\mathrm{C}_{9} \mathrm{H}_{13} \mathrm{ClO}_{2}\left(\mathrm{M}^{+}\right)$188.0604, found 188.0599 .

endo-1-(7-Chlorobicyclo[4.1.0]hept-7-yl)-3-buten-1-one (4g) (trans-adduct)

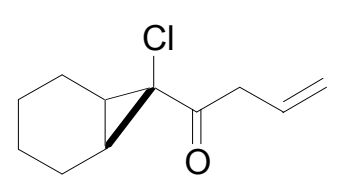

$4 \mathrm{~g}$

Following the procedure of method III, the reaction of $1 \mathrm{~g}(165 \mathrm{mg}, 1.00 \mathrm{mmol})$ gave the product $4 \mathrm{~g}(73 \mathrm{mg}, 55 \%)$. Following the procedure of method IV, the reaction of 11 (210 mg, $1.00 \mathrm{mmol})$ gave the product $\mathbf{4 g}(126 \mathrm{mg}, 64 \%)$. 4g: colorless oil; ${ }^{1} \mathrm{H}$ NMR (400 MHz, $\left.\mathrm{CDCl}_{3}\right) \delta$ 1.21-1.30 (m, 2H), 1.35-1.44 (m, 2H), 1.58-1.64(m, 2H), 1.90-2.00 (m, 4H), 3.62-3.64 (m, 2H), 5.10-5.20 (m, 2H), 5.88-5.98 (m, $1 \mathrm{H}) ;{ }^{13} \mathrm{C}$ NMR $\left(100 \mathrm{MHz}, \mathrm{CDCl}_{3}\right) \delta 18.8,20.8,27.4,44.9,58.5,118.5,130.8,205.6$; IR (neat) 2931, 1693, $1639 \mathrm{~cm}^{-1}$; HRMS (EI) calcd for $\mathrm{C}_{11} \mathrm{H}_{15} \mathrm{ClO}\left(\mathrm{M}^{+}\right)$198.0811, found 198.0818.

In order to determine the relative configuration, aldehyde $\mathbf{2 g}$ (trans-adduct) which was stereoselectively prepared by the formylation converted into ketone $\mathbf{4 a}$ in two steps [(1) $\left(\mathrm{CH}_{2}=\mathrm{CHCH}_{2}\right) \mathrm{MgBr}$, (2) Jones' oxidation]. Thus, the relative configuration of $4 \mathbf{a}$ was assigned as trans-adduct.
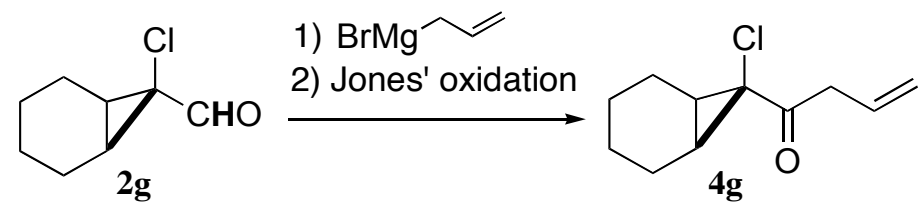

endo-1-(9-Chlorobicyclo[6.1.0]non-9-yl)-3-buten-1-one (4h) (trans-adduct)

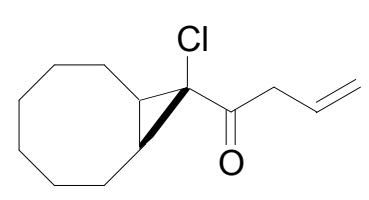

4h

Following the procedure of method III, the reaction of $\mathbf{1 h}(193 \mathrm{mg}, 1.00$ mmol) gave the product $\mathbf{4 g}(109 \mathrm{mg}, 48 \%)$. Following the procedure of method IV, the reaction of $\mathbf{1} \mathbf{m}(238 \mathrm{mg}, 1.00 \mathrm{mmol})$ gave the product $\mathbf{4 h}$ (123 $\mathrm{mg}, 54 \%$ ). Based on the analogy of spectra data of $\mathbf{4 g}$, the relative 
configuration of $\mathbf{4 h}$ was determined as trans-adduct. $\mathbf{4 h}$ : colorless oil; ${ }^{1} \mathrm{H}$ NMR $\left(400 \mathrm{MHz}, \mathrm{CDCl}_{3}\right)$ ठ 1.32-1.51, (m, 6H), 1.58-1.72 (m, 6H), 1.80-1.85 (m, 2H), $3.62(\mathrm{dt}, J=1.5,6.8 \mathrm{~Hz}, 2 \mathrm{H}), 5.10-5.20$ (m, 2H), 5.89-6.00 (m, 1H); ${ }^{13} \mathrm{C}$ NMR (100 MHz, $\left.\mathrm{CDCl}_{3}\right) \delta$ 22.7, 26.4, 28.2, 33.5, 45.0, 56.9, 118.5, 130.7, 205.7; IR (neat) 2923, 1697, $1643 \mathrm{~cm}^{-1}$; HRMS (EI) calcd for $\mathrm{C}_{13} \mathrm{H}_{19} \mathrm{ClO}\left(\mathrm{M}^{+}\right) 226.1124$, found 226.1128 .

\section{endo-1-(7-Bromobicyclo[4.1.0]hept-7-yl)-3-buten-1-one (4i) (trans-adduct)}

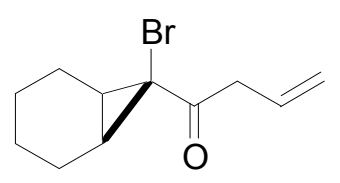

$4 \mathbf{i}$ Following the procedure of method IV, the reaction of $1 \mathbf{i}(253 \mathrm{mg}, 1.00 \mathrm{mmol})$ gave the product $4 \mathbf{i}(42 \mathrm{mg}, 17 \%)$. Based on the analogy of spectra data of $\mathbf{4 g}$, the relative configuration of $\mathbf{4 i}$ was determined as trans-adduct. $4 \mathbf{i}$ : colorless oil; ${ }^{1} \mathrm{H}$ NMR (400 MHz, $\left.\mathrm{CDCl}_{3}\right) \delta$ 1.32-1.50 (m, 4H), 1.58-1.72 (m, 4H), 1.80$1.85(\mathrm{~m}, 2 \mathrm{H}), 3.61-3.62(\mathrm{~m}, 1 \mathrm{H}), 3.63-3.64(\mathrm{~m}, 1 \mathrm{H}), 5.11-5.20(\mathrm{~m}, 2 \mathrm{H}), 5.90-6.00(\mathrm{~m}, 1 \mathrm{H}) ;{ }^{13} \mathrm{C}$ NMR (100 MHz, $\left.\mathrm{CDCl}_{3}\right) \delta 18.8,20.8,27.4,44.9,52.8,118.5,130.8,203$; IR (neat) 2931, 1693, $1639 \mathrm{~cm}^{-1}$; HRMS (EI) calcd for $\mathrm{C}_{11} \mathrm{H}_{15} \mathrm{BrO} 242.0306$, found 242.0312.

$\left(1 R^{*}, 2 R^{*}, 5 S^{*}, 6 R^{*}\right)-$ and $\left(1 R^{*}, 2 S^{*}, 5 S^{*}, 6 R^{*}\right)-2$-Allyl-1-chloro-5-methyl-6-phenyl-3-oxabicyclo[3.1.0]hexan-2ol (5o)

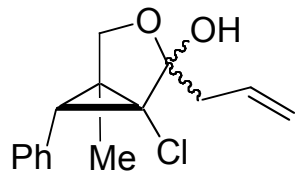

50

Following the procedure of method IV, the reaction of $10(100 \mathrm{mg}, 0.36 \mathrm{mmol})$ gave the product $50(34 \mathrm{mg}, 35 \%)$. A couple of diastereoisomeric lactols apparently existed (ratio $=2 / 1$ in $\mathrm{CDCl}_{3}$ ). Based on the analogy of spectra data of lactol $\mathbf{3 f}$, the relative configuration of $\mathbf{5 0}$ was tentatively determined as cisadduct (lactol). 5o: colorless oil; ${ }^{1} \mathrm{H}$ NMR $\left(400 \mathrm{MHz}, \mathrm{CDCl}_{3}\right) \delta 1.33$ (s, 3H x 2/3), $1.54(\mathrm{~s}, 3 \mathrm{H} \times 1 / 3), 2.13-2.20(\mathrm{~m}, 1 \mathrm{H} \times 2 / 3), 2.35(\mathrm{~s}, 1 \mathrm{H}), 2.49-2.53(\mathrm{~m}, 1 \mathrm{H} \times 1 / 3), 2.57-2.62(\mathrm{~m}$, $1 \mathrm{H} \times 1 / 3), 2.65-2.71(\mathrm{~m}, 1 \mathrm{H} \times 2 / 3), 2.81(\mathrm{~s}, 1 \mathrm{H} \times 1 / 3), 3.02(\mathrm{~s}, 1 \mathrm{H} \times 2 / 3), 3.69(\mathrm{~d}, J=8.4 \mathrm{~Hz}, 1 \mathrm{H} \times$ 1/3), $3.87(\mathrm{~d}, J=8.4 \mathrm{~Hz}, 1 \mathrm{H} \times 1 / 3), 3.99(\mathrm{~d}, J=8.8 \mathrm{~Hz}, 1 \mathrm{H} \times 2 / 3), 4.18(\mathrm{~d}, J=8.8 \mathrm{~Hz}, 1 \mathrm{H} \times 1 / 3)$, 5.03-5.10 (m, $2 \mathrm{H}$ x 2/3), 5.22-5.28 (m, $2 \mathrm{H}$ x 1/3), 5.82-5.99 (m, $1 \mathrm{H}) 7.23-7.39(\mathrm{~m}, 5 \mathrm{H}) ;{ }^{13} \mathrm{C} \mathrm{NMR}$ $\left(100 \mathrm{MHz}, \mathrm{CDCl}_{3}\right) \delta 13.5,14.7,23.4,26.5,30.5,35.4,36.0,40.0,53.8,71.4,72.9,116.9,120.4$, 127.6, 127,6, 128.3, 128.3, 128.8, 128.9, 131.7, 134.4, 134.6; IR (neat) 3419, 1643, 1382, $910 \mathrm{~cm}^{-1}$; HRMS (EI) calcd for $\mathrm{C}_{15} \mathrm{H}_{17} \mathrm{ClO}_{2}\left(\mathrm{M}^{+}\right)$264.0917, found 264.0913.

\section{exo-7-Bromobicyclo[4.1.0]heptane-7-carbaldehyde (14i) (cis-adduct)}

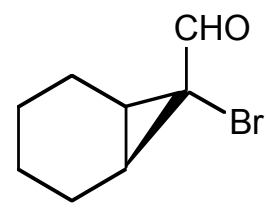

$14 \mathbf{i}$

(Method A) BuLi (1.52 $\mathrm{M}$ in hexane, $3.62 \mathrm{~mL}, 5.50 \mathrm{mmol}$ ) was added to a stirred solution of 7,7-dibromobicyclo[4.1.0]heptane (1.27 g, $5.00 \mathrm{mmol})$ in THF $(10 \mathrm{~mL})$ at $-100{ }^{\circ} \mathrm{C}$ under an Ar atmosphere, followed by being stirred at the same temperature for $30 \mathrm{~min}$. DMF $(2.50 \mathrm{~mL}, 32.3 \mathrm{mmol})$ was added to the mixture at the same temperature, which was stirred for $1 \mathrm{~h}$. Water was added to the mixture, which was extracted three times with $\mathrm{Et}_{2} \mathrm{O}$. The combined organic phase was washed with water, 
brine, dried $\left(\mathrm{Na}_{2} \mathrm{SO}_{4}\right)$, and concentrated. The obtained crude oil was purified by $\mathrm{SiO}_{2}$-column chromatography (hexane $\left./ \mathrm{Et}_{2} \mathrm{O}=50 / 1\right)$ to give the desired product $14 \mathbf{i}(705 \mathrm{mg}, 65 \%)$. The relative configuration of aldehyde 14i was determined as cis-adduct by the comparison of chemical shift $(\mathrm{CHO})$ of $\mathbf{2} \mathbf{i}$ (another stereoisomer) with that of 14i. Based on the deshielding effect of alkyl group, the chemical shift (CHO: $9.55 \mathrm{ppm}$ ) of aldehyde 14i was assigned to that of cis-adduct. 14i: pale yellow oil; ${ }^{1} \mathrm{H}$ NMR (300 MHz, $\left.\mathrm{CDCl}_{3}\right) \delta$ 1.33-1.56 (m, 4H), 1.77-1.92 (m, 2H), 1.95-2.11 (m, 2H), 2.17-2.30 (m, 2H), $9.55(\mathrm{~s}, 1 \mathrm{H}) ;{ }^{13} \mathrm{C}$ NMR (75 MHz, $\left.\mathrm{CDCl}_{3}\right) \delta$ 18.7, 20.9, 32.6, 44.1, 195.4; IR (KBr) 2936, 1707, 1447, 1283, 1263, 1181, 1125, 893, $777 \mathrm{~cm}^{-1}$; HRMS (EI) calcd for $\mathrm{C}_{8} \mathrm{H}_{11} \mathrm{BrO}$ $\left(\mathrm{M}^{+}\right)$201.9993, found 201.9991 .

\section{Methyl exo-7-bromobicyclo[4.1.0]heptane-7-carboxylate (14ii) (cis-adduct)}

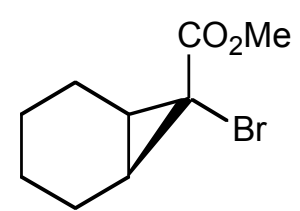

14ii

(Method B) In a similar manner using $\mathrm{CO}_{2}$ (dry ice; c.a. $2.5 \mathrm{~g}$ ) instead of DMF for the preparation of $\mathbf{1 4} \mathbf{i}$, the mixture was quenched with water, which was extracted twice with $\mathrm{Et}_{2} \mathrm{O}$. Separated aqueous layer was acidified using $6 \mathrm{M}-\mathrm{HCl}$ aqueous solution, which was re-extracted three times with $\mathrm{Et}_{2} \mathrm{O}$. The combined organic phase was washed with water and brine, dried $\left(\mathrm{Na}_{2} \mathrm{SO}_{4}\right)$ and concentrated. The obtained crude carboxylic acid (solid) was used for the next step without purification. MeI $(747 \mu \mathrm{L}, 12.0$ mmol) was added to a stirred solution of the carboxylic acid $(1.10 \mathrm{~g}, 5.00 \mathrm{mmol})$ and $\mathrm{K}_{2} \mathrm{CO}_{3}(1.66 \mathrm{~g}$, $12.0 \mathrm{mmol})$ in DMF $(5.0 \mathrm{~mL})$ at $20-25{ }^{\circ} \mathrm{C}$ under an Ar atmosphere, followed by being stirred at same temperature for $1 \mathrm{~h}$. The mixture was quenched with water, which was extracted three times with $\mathrm{Et}_{2} \mathrm{O}$. The combined organic phase was washed with water, brine, dried $\left(\mathrm{Na}_{2} \mathrm{SO}_{4}\right)$, and concentrated. The obtained crude oil was purified by $\mathrm{SiO}_{2}$-column chromatography (hexane/AcOEt $=60 / 1)$ to give the desired product $14 \mathrm{ii}(860 \mathrm{mg}, 64 \%)$. In order to determine the relative configuration, ester 14ii derived from aldehyde 14i (cis-adduct) [2 steps: (1) Jones' oxidation (2) methylation]. Thus, the relative configuration of 14ii was assigned as cis-adduct. 14ii: pale yellow oil; ${ }^{1} \mathrm{H}$ NMR (300 MHz, $\left.\mathrm{CDCl}_{3}\right) \delta$ 0.97-1.11 (m, 2H), 1.14-1.34 (m, 2H), 1.65-1.78 (m, 2H), 1.82$1.98(\mathrm{~m}, 4 \mathrm{H}), 3.79$ (s, 3H); ${ }^{13} \mathrm{C}$ NMR (75 MHz, $\left.\mathrm{CDCl}_{3}\right) \delta$ 19.3, 20.4, 25.1, 31.8, 52.6, 167.9; IR (neat) 3007, 2948, 2870, 1734, 1437, 1319, 1273, 1204, $1101 \mathrm{~cm}^{-1}$; HRMS (EI) calcd for $\mathrm{C}_{9} \mathrm{H}_{13} \mathrm{BrO}_{2}$ $\left(\mathrm{M}^{+}\right)$232.0099, found 232.0108.

exo-7-Chlorobicyclo[4.1.0]heptane-7-carbaldehyde (14l) (cis-adduct)

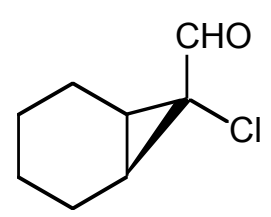

141

Following the procedure of method A, the reaction of 7-bromo-7chlorobicyclo[4.1.0]heptane $(2.10 \mathrm{~g}, 10.0 \mathrm{mmol})$ with ${ }^{n} \mathrm{BuLi}(1.52 \mathrm{M}$ in hexane, $7.23 \mathrm{~mL}, 11.0 \mathrm{mmol})$ and DMF $(5.00 \mathrm{~mL}, 64.6 \mathrm{mmol})$ gave the desired product $14 \mathrm{l}$ (675 mg, 43\%). Relative structure of $\mathbf{1 4 1}$ was determined based on the comparison between NMR spectrum of $\mathbf{2 g}$ and $\mathbf{1 4 l}$. The relative configuration of aldehyde $\mathbf{1 4 l}$ was determined as cis-adduct by the comparison of chemical shift $(\mathrm{CHO})$ of $\mathbf{1 4 l}$ with that of $\mathbf{2} \mathbf{g}$ (another 
stereoisomer). Based on the deshielding effect of alkyl group, the chemical shift (CHO $9.72 \mathrm{ppm}$ ) of aldehyde 14l was assigned to that of $c i s$-adduct. 14l: pale yellow oil; ${ }^{1} \mathrm{H} \mathrm{NMR}\left(300 \mathrm{MHz}, \mathrm{CDCl}_{3}\right) \delta$ 1.34-1.57 (m, 4H), 1.75-1.88 (m, 2H), 1.94-2.09 (m, 2H), 2.11-2.19 (m, 2H), $9.72(\mathrm{~s}, 1 \mathrm{H}) ;{ }^{13} \mathrm{C} \mathrm{NMR}$ $\left(75 \mathrm{MHz}, \mathrm{CDCl}_{3}\right) \delta 18.3,20.8,33.0,53.2,196.1$; IR (neat) 2940, 2861, 1713, 1447, 1287, 1267, 1186, 1128, 982, $631 \mathrm{~cm}^{-1}$; HRMS (EI) calcd for $\mathrm{C}_{8} \mathrm{H}_{11} \mathrm{ClO}\left(\mathrm{M}^{+}\right)$158.0498, found 158.0503 .

\section{Methyl exo-7-chlorobicyclo[4.1.0]heptane-7-carboxylate (14ll) (cis-adduct)}

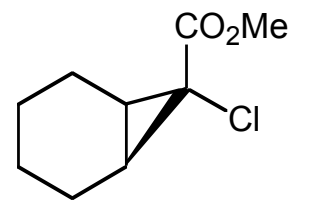

1411

Following the procedure of method $\mathrm{B}$, the reaction of 7-bromo-7chlorobicyclo[4.1.0]heptane $(2.10 \mathrm{~g}, 10.0 \mathrm{mmol})$ with ${ }^{n} \mathrm{BuLi}(1.52 \mathrm{M}$ in hexane, $7.23 \mathrm{~mL}, 11.0 \mathrm{mmol}$ ) and $\mathrm{CO}_{2}$ (dry ice; c.a. $5.0 \mathrm{~g}$ ) gave the crude solid (654 mg, $3.75 \mathrm{mmol})$. The reaction of crude solid with $\mathrm{K}_{2} \mathrm{CO}_{3}(1.24 \mathrm{~g}, 9.00 \mathrm{mmol})$ and MeI (570 $\mu \mathrm{L}, 9.00 \mathrm{mmol})$ gave the product $141 \mathrm{l}(334 \mathrm{mg}, 47 \%)$. In order to determine the relative configuration, ester 1411 derived from aldehyde 141 (cis-adduct) [2 steps: (1) Jones' oxidation (2) methylation]. Thus, the relative configuration of $\mathbf{1 4 l l}$ was assigned as cis-adduct. 14ll: pale yellow oil; ${ }^{1} \mathrm{H}$ NMR (300MHz, $\left.\mathrm{CDCl}_{3}\right) \delta$ 1.07-1.36 (m, 4H), $\delta$ 1.68-1.74 (m, 2H), 1.75-1.99 (m, 4H), 3.80 $(\mathrm{m}, 3 \mathrm{H}) ;{ }^{13} \mathrm{C}$ NMR $(75 \mathrm{MHz}, \mathrm{CDCl} 3) \delta$ 18.9, 20.4, 26.3, 44.2, 52.6, 168.0; IR (neat) 2940, 2861, 1738, 1437, 1321, 1206, 1165, $1103 \mathrm{~cm}^{-1}$; HRMS (EI) calcd for $\mathrm{C}_{9} \mathrm{H}_{13} \mathrm{ClO}_{2}\left(\mathrm{M}^{+}\right)$188.0604, found 188.0607 .

\section{$\left(1 R^{*}, 2 S^{*}\right)-1-B r o m o-2-p h e n y l c y c l o p r o p a n e-1-c a r b a l d e h y d e ~(14 p)$ (cis-adduct)}

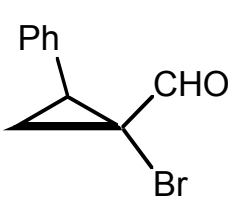

$14 p$ Following the procedure of method A, the reaction of 1,1-dibromo-2phenylcyclopropane $(2.10 \mathrm{~g}, 10.0 \mathrm{mmol})$ with DMF $(5.00 \mathrm{~mL}, 64.6 \mathrm{mmol})$ and ${ }^{n} \mathrm{BuLi}(1.52 \mathrm{M}$ in hexane, $7.23 \mathrm{~mL}, 11.0 \mathrm{mmol})$ gave the product $14 \mathrm{p}(675 \mathrm{mg}$, $64 \%$ ). Based on the analogy of spectra data of $\mathbf{3 a}$, the relative configuration of $\mathbf{1 4} \mathbf{p}$ was determined as cis-adduct. 14p: pale yellow oil; ${ }^{1} \mathrm{H}$ NMR $\left(300 \mathrm{MHz}, \mathrm{CDCl}_{3}\right) \delta 1.93$ (exo, dd, $J$ $=6.2 \mathrm{~Hz}, J=8.6 \mathrm{~Hz}, 0.05 \mathrm{H}$ ), 2.05 (endo, dd, $J=6.9 \mathrm{~Hz}, J=9.6 \mathrm{~Hz}, 0.95 \mathrm{H}$ ), 2.27 (exo, dd, $J=6.2$ $\mathrm{Hz}, J=10.3 \mathrm{~Hz}, 0.05 \mathrm{H}), 2.45$ (endo, dd, $J=6.9 \mathrm{~Hz}, J=8.3 \mathrm{~Hz}, 0.95 \mathrm{H}), 2.91$ (exo, t, $J=9.3 \mathrm{~Hz}$, $0.05 \mathrm{H}), 3.33$ (endo, $\mathrm{t}, J=9.3 \mathrm{~Hz}, 0.95 \mathrm{H}), 7.21-7.39(\mathrm{~m}, 5 \mathrm{H}), 8.70(\mathrm{~s}, 1 \mathrm{H}) ;{ }^{13} \mathrm{C} \mathrm{NMR}(75 \mathrm{MHz}$, $\left.\mathrm{CDCl}_{3}\right) \delta 22.0,38.3,40.3,127.8,128.6,128.6,133.2$, 193.5; IR (neat) 3030, 2838, 1715, 1497, 1451, 1263, 1200, 926, 889, $698 \mathrm{~cm}^{-1}$; HRMS (EI) calcd for $\mathrm{C}_{10} \mathrm{H}_{9} \mathrm{BrO}\left(\mathrm{M}^{+}\right)$223.9873, found 223.9875 .

\section{$\left(1 R^{*}, 2 S^{*}\right)$-Methyl-1-bromo-2-phenylcyclopropane-1-carboxlate (14pp) (cis-adduct)}

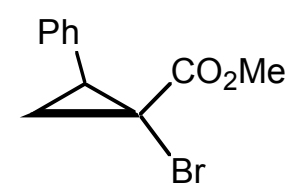

$14 p p$

Following the procedure of method $\mathrm{B}$, the reaction of 1,1-dibromo-2phenylcyclopropane $(1.10 \mathrm{~g}, 4.00 \mathrm{mmol})$ with ${ }^{n} \mathrm{BuLi}(1.52 \mathrm{M}$ in hexane, $2.89 \mathrm{~mL}$, $4.40 \mathrm{mmol}$ ) and $\mathrm{CO}_{2}$ (dry ice; c.a. $2.0 \mathrm{~g}$ ) gave the crude solid (866 mg, 3.60 mmol). The reaction of crude solid with $\mathrm{K}_{2} \mathrm{CO}_{3}(1.20 \mathrm{~g}, 8.64 \mathrm{mmol})$ and MeI 
$(537 \mu \mathrm{L}, 8.64 \mathrm{mmol})$ gave the desired product 14pp (582 mg, 57\%). 14pp: pale yellow oil; ${ }^{1} \mathrm{H}$ NMR $\left(300 \mathrm{MHz}, \mathrm{CDCl}_{3}\right) \delta 1.79(\mathrm{dd}, J=6.5 \mathrm{~Hz}, J=10.0 \mathrm{~Hz}, 1 \mathrm{H}), 2.37$ (dd, $J=6.5 \mathrm{~Hz}, J=8.6 \mathrm{~Hz}$, $1 \mathrm{H}), 3.07(\mathrm{t}, J=9.3 \mathrm{~Hz}, 1 \mathrm{H}), 3.42(\mathrm{~s}, 3 \mathrm{H}), 7.19-7.33(\mathrm{~m}, 5 \mathrm{H}) ;{ }^{13} \mathrm{C} \mathrm{NMR}\left(75 \mathrm{MHz}, \mathrm{CDCl}_{3}\right) \delta 22.1$, $31.3,36.7,52.9,127.3,128.1,128.6,134.3,167.3$; IR (neat) 3030, 2953, 1738, 1499, 1437, 1314, 1217, 1121, 1069, $698 \mathrm{~cm}^{-1}$; HRMS (EI) calcd for $\mathrm{C}_{11} \mathrm{H}_{11} \mathrm{BrO}_{2}\left(\mathrm{M}^{+}\right)$253.9942, found 253.9944 .

\section{$\left(1 R^{*}, 2 R^{*}\right)$-Methyl -1-bromo-2-butylcyclopropane-1-carboxylate (14qq) (cis-adduct)}

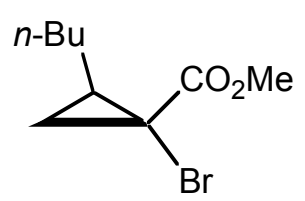

$14 q q$

Following the procedure of method $\mathrm{B}$, the reaction of 1,1-dibromo-2butylpropane (512 mg, $2.00 \mathrm{mmol}$ ) with ${ }^{n} \mathrm{BuLi}$ (1.52 $\mathrm{M}$ in hexane, $1.45 \mathrm{ml}, 2.20$ $\mathrm{mmol}$ ) and $\mathrm{CO}_{2}$ (dry ice; c.a. $1.0 \mathrm{~g}$ ) gave the crude solid (224 $\mathrm{mg}, 1.10 \mathrm{mmol}$ ). The reaction of crude solid with $\mathrm{K}_{2} \mathrm{CO}_{3}(365 \mathrm{mg}, 2.64 \mathrm{mmol})$ and $\mathrm{MeI}(164 \mu \mathrm{L}$, $2.64 \mathrm{mmol}$ ) gave the desired product 14qq (160 mg, 36\%). 14qq: pale yellow oil; ${ }^{1} \mathrm{H}$ NMR (300 $\left.\mathrm{MHz}, \mathrm{CDCl}_{3}\right) \delta 0.89(\mathrm{t}, J=7.2 \mathrm{~Hz}, 3 \mathrm{H}), 1.22-1.38(\mathrm{~m}, 4 \mathrm{H}), 1.43-1.52(\mathrm{~m}, 3 \mathrm{H}), 1.62(\mathrm{dd}, J=6.2 \mathrm{~Hz}$, $J=8.3 \mathrm{~Hz}, 1 \mathrm{H}), 1.71-1.84(\mathrm{~m}, 1 \mathrm{H}), 3.77(\mathrm{~s}, 3 \mathrm{H}) ;{ }^{13} \mathrm{C} \mathrm{NMR}\left(75 \mathrm{MHz}, \mathrm{CDCl}_{3}\right) \delta 13.9,22.2,24.7$, 27.3, 29.7, 31.0, 34.1, 53.2, 169.3; IR (neat) 2957, 2930, 2863, 1725, 1437, 1316, 1209, $1127 \mathrm{~cm}^{-1}$; HRMS (EI) calcd for $\mathrm{C}_{9} \mathrm{H}_{15} \mathrm{BrO}_{2}\left(\mathrm{M}^{+}\right)$234.0255, found 234.0261. 